\title{
OPPORTUNITIES AND WORKING CONDITIONS
}

\author{
ELIZABETH GRIFFIN \\ Institute of Astronomy \\ University of Cambridge \\ The Observatories \\ Madingley Road \\ Cambridge CBз OHA, UK
}

It is impossible to pose, explain and solve in a mere 15 minutes a complex puzzle like this that has been with us for centuries. There are so many different facets; it is hard to focus on a problem that has no nucleus and to summarize a situation that has neither beginning nor end, and some of you are therefore bound to disagree with at least some of what I say.

I singled out the three questions, "Is there a problem?", "Is there need for improvement?" and "What are the possible ways to improve the situation?", because each seems to beg the next and all three can therefore be tackled together. The official answer to the first question is "No, there is no discrimination, and no Problem", because the large majority of institutions declare themselves Equal Opportunity Employers, and statistics show that women are usually appointed to positions in at least the same proportion, relative to their male peers, as they apply. The nagging worry is why they do not feel able to apply in the sort of numbers that reflect their population. This is more than a social problem; anyone who counts the cost of educating his or her daughter to the age of 25 will know that it has an economic side too. And the reality of the Problem? I attended the 1992 Baltimore meeting on "Women in Astronomy", and for the first of the two days the 175 or so women in the 200 -strong audience simply let off steam, flung mud, aired grievances, and demonstrated that a Problem was very much in evidence; everyone had one, in some disguise - except, possibly, those who occupied the seats on the Panel the next day and answered the political questions. But put 175 men together in a free-for-all, and they would probably discuss .... maybe how best to spend the next $\$$ M100 on space astronomy. Why the difference? Because, notwithstanding Equal Opportunities, the platforms from which these two sects operate are 
very Unequal. Consider two colleagues with comparably mature careers, one supported by the institution, the other by 'soft' money. However successfully the grant funding may have been renewed every three years or so, the fact remains that while one can look back over a well-lived academic existence, the other can claim only a refusal to die.

What exactly is the nature of the Problem? In plain words, women feel uncomfortable in a world planned and ordained the way it is. Why? Sheila Tobias put it succinctly: "What men do is good because it is men that do it." Many women labour under the stigma of the converse: "What women do is only worthy of being done by ...", and thereby deny themselves almost consciously the very opportunities which they wish to have had. Women have less personal power in their lives; more emotional demands are made upon them, more pressure is put on them to be conformist, to put up with things, to act as the 'glue' that holds society together, and they receive inadequate recognition for those efforts. Their pattern for living has been written by cultural traditions stretching back for aeons, and to turn deliberately against all this by choosing to follow a career that is pretty merciless when it comes to opting out for even a small number of those prime years requires a disproportionately large supply of courage and energy. Put like that, it is hardly surprising that far fewer women than men, from my cultural background at least, actually feel that a subject like Astronomy is unequivocally inviting. The atmosphere does not invite, the prospects do not invite, society does not invite (however much its government claims to need more women scientists), and the rôle models (what few there are) all have their versions of how they overcame their gender's incipient Problem, or what has been aptly called "the invisible obstacle race".

That tenacity and determination to succeed is an environmentally-induced characteristic if ever there was one, but it demonstrates a force that could be harnessed. Because human thoughts and feelings are inextricably linked the situation has to be handled dispassionately, and that is not easy when every member of the scientific professional society is by definition either a defender or an upholder of some attitude. Nothing can be moved without change, but change is usually unwelcome; it runs counter to human nature, because the status quo provides a sense of security and predictability whereas change, even when for the better, is unsettling.

But where the memory is long (and mine dates back over 30 years in Cambridge) we can identify changes that are in the wind. My University is actually doing something positive to help the young, and even the not so young, women tackle their Problems: it is implementing a professional programme called Springboard, which is a course of four Workshops designed to help women develop their potential by recognizing and increasing their confidence, gaining courage and acquiring the nicer grace of assertion. The 
programme is available in some other countries, I know, and it would be worth making enquiries if you think it could be useful in your own institution. It may not work miracles overnight, but it does provide a catalyst for changes in attitude and outlook.

But perhaps the real question to answer is, "Is there (literally) room for improvement?" I think there needs to be a subtle change (that difficult concept again, but it really is unavoidable) in the perception of what constitutes a truly productive research team, in a way that poses no threats and sheds no blood. More thought must be given to the value of complementary in a research project, not just the end results but the manner of their achievement and the quality of the associated team-work. The special attributes, including lateral thinking and multi-tasking as well as the more obvious ones, which women possess (either innately or environmentallyinduced, the evidence is mixed) are such that, without them, no team will survive healthily for long. In order for a living plant to flourish all features must receive fair attention, roots as well as flowers, the supportive as well as the showy. A similar philosophy applied to other living organizations like scientific research teams will benefit the participants, the results, the sponsors and ultimately the society. 